\title{
VARIAÇÕES DIRECIONAIS NOS PARÂMETROS GRANULOMÉTRICOS: UM INDICADOR APROPRIADO PARA O SENTIDO DA DERIVA LITORÂNEA
}

\author{
ABÍLIO C.S.P. BITTENCOURT*, GERALDO S.V. BOAS* e FÉLIX F. FARIAS**
}

\begin{abstract}
DIRECTIONAL CHANGES IN GRAIN SIZE PARAMETERS: AN APPROPRIATE INDICATOR FOR THE LONGSHORE TRANSPORT DIRECTION. The method of analysis adopted in thi paper is based on a model, proposed by McLaren (1981), according to which during transport, changes in sediment characteristics (mean grain size, standard deviation and skewness) follow indentifiable trends from the source to the final depositional site. Once established, these trends in grain size parameters may indicate the sediment transport path. Three beaches were studied showing distinct beach dynamics. A great number of sediment samples were col lected at these three beaches, during different periods of time. The results have shown that in $74 \%$ of the cases the changes in grain size parameters of the Caixa-Pregos beach (Itaparica Island-B A), $68 \%$ atthe Armação beach (Salvador-B A) and $66 \%$ at the Atalaiabeach (LuisCorreia-PI) have coincided with the longshore drift direction at these beaches. Atthe Atalaia beach however, during the dry season, when strong onshore winds remove sand from the beach face, only in $25 \%$ of the cases the changes in grain size parameters coincided with the longshore drift direction. In this case, the discrepancy is attributed to the fact that eolian deflation is non-uniform along the entire beach length. Anomalous results, or those showing no trends at all, obtained during some days, are probably related to errors associated with sampling. This metodology can be possibly applied to determine longshore drift direction in the rock record if sampling is conducted along the depositional strike and the samples are collected on the same depositional surface.
\end{abstract}

Keywords: Longshore drift, grain-size parameters, transport direction.

\begin{abstract}
RESUMO O método de análise adotado neste trabalho é baseado em um modelo proposto por McLaren (1981), segundo o qual, durante o transporte, as mudanças nas características do sedimento (média, desvio padrão e assimetria) seguem tendências identificáveis desde afonte até o depósito. Uma vez estabelecidas, essas tendências nos parâmetros granulométricos podem indicar a trajetória de transporte do sedimento. Três praias foram escudadas, mostrando diferentes dinâmicas praiais. Um grande número de amostras de sedimento foi coletado nessas três praias, durante diferentes períodos de tempo. Os resultados mostraram que $74 \%$ dos casos de mudanças nos parâmetros granulométricos na praia de Caixa-Pregos (Ilha de Itaparica - BA), 68\% na de Armação (Salvador - BA) e 66\% na de Atalaia (Luis Correia- PI) coincidiram como sentido da deriva litorânea atuante nessas praias. Na praia de Atalaia, durante a estação seca, quando fortes ventos soprando continente adentro removem are ia da face da praia, somente em $25 \%$ dos casos as mudanças nos parâmetros granulométricos coincidiram com o sentido da deriva 1 itorânea nela atuante. Nesse caso, tal discrepância é atribuída ao fato de a deflação eólica não ser uniforme ao longo de toda a praia. Resultados anômalos, ou sem nenhuma indicação, obtidos em alguns dias, estão provavelmente relacionados a erros associados com a amostragem. Esta metodologia pode, possivelmente, ser aplicada para determinar o sentido da deriva litorânea no registro estratigráfico, desde que a amostragem seja conduzida ao longo da direção deposicional e as amostras sejam coletadas na mesmasuperfície deposicional.
\end{abstract}

Palavras-chaves: Deriva 1 itorânea, parâmetros granulométricos, sentido de transporte.

INTRODUÇ̃̃O Segundo McLaren (1981), as características de um depósito sedimentar, tais como a média, o desvio padrão e a assimetria, são herdadas de sua fonte e são dependentes de processos do tipo a. joeiramento, b. deposição seletiva ou parcial da distribuição granulométrica em transporte e c. deposição total da distribuição granulométrica em transporte. Desse modo, segundo ele, as mudanças na distribuição granulométrica seguem tendências claramente identificáveis desde a fonte até o depósito. Uma vez estabelecidas, essas tendências indicam uma trajetória de transporte para a movimentação do sedimento que pode sugerir um modelo que identifique o ambiente de deposição. Considerando uma fonte sedimentar hipotética, McLaren (1981), dedutivamente, chegou à conclusão de que os mecanismos pelos quais um depósito pode vir a ser derivado dessa fonte podem ser ilustrados em três casos distintos. Ademais, para cada um dos casos, McLaren (1981) demonstra as mudanças relativas que devem ocorrer nos parâmetros estatísticos escolhidos (média, desvio padrão e assimetria) entre a fonte sedimentar hipotética e o depósito resultante. Segundo esse autor, entre esses casos, o mais provável a vir a ocorrer na sedimentação na face da praia, que é o objeto de estudo deste trabalho, está relacionado a uma deposição seletiva do sedimento em transporte, como uma resposta à contínua movimentação que os sedimentos desse ambiente estão sujeitos pela ação da saca e ressaca.

Este trabalho objetivou aplicar o método de análise de tendência desenvolvido por McLaren (1981) em três praias que apresentam dinâmicas de sedimentação bem distintas entre si, visando verificar a sua aplicabilidade na definição do sentido de transporte de sedimentos. Essas praias foram escolhidas porque já têm bem definidos os seus padrões de transporte de sedimentos na face da praia. Ao invés de se ater a medidas efetuadas em amostras colhidas na face da praia em apenas um único dia, como feito por McLaren (1981), este trabalho apresenta dados relativos a uma série de amostras coletadas ao longo de um certo período de tempo em cada praia, alcançando as diferentes fases do ciclo praial, que apresentam características morfodinâmicas bem diferentes entre si. Essas praias, denominadas de Armação (Salvador Bahia), Atalaia (Luís Correia - Piauí) e Caixa - Pregos (Baía de Todos os Santos - Bahia), tiveram a sua dinâmica de 
sedimentação definida, no caso da praia de Armação, por Farias et al. (1985) e Bittencourt et al. (1987) e, quanto às duas últimas, respectivamente por Bittencourt et al. (1990a) e Bittencourt et al. (1990b). Nas três praias, as amostras utilizadas foram coletadas longitudinalmente às mesmas, na face da praia superior, sendo representativas dos primeiros dois centímetros superficiais do sedimento praial. As amostras foram quarteadas e, após a eliminação dos sais solúveis de uma quantidade em torno de $50 \mathrm{~g}$, peneiradas a seco com Rotap, através de um conjunto de peneiras com intervalo de $1 / 2 \mathrm{~F}$, sendo então as frações retidas em cada peneira, pesadas em balança de precisão Mettler até a segunda casa decimal. Posteriormente, para as praias de Armação (Bittencourt et al. 1987) e Atalaia (O. Moita $\mathrm{F}^{\circ}$, comunicação pessoal), os valores da média, desvio padrão e assimetria foram calculados segundo Folk \& Ward (1957); para a praia de CaixaPregos (F.F. Farias, comunicação pessoal), segundo Friedman (1961), com precisão de até a segunda casa decimal. Por fim, para cada dia em que foram coletadas amostras, foram comparados, individualmente por cada praia, os valores do desvio padrão, da assimetria e da média, de cada amostra com todas as demais, sendo identificandos os casos que se enquadram no modelo proposto por McLaren (1981) para a sedimentação na face da praia. Segundo esse modelo, no sentido do transporte, uma amostra considerada como depósito deverá ter melhor selecionamento e ser mais positivamente assimétrica em relação às que a antecedem - as quais são consideradas como fonte - enquanto a média poderá ser mais fina, igual ou mais grossa.

DINÂMICA SEDIMENTAR DAS PRAIAS DE ARMAÇÃO, ATALAIA E CAIXA - PREGOS A seguir será feita uma descrição sumária das principais características da dinâmica sedimentar das praias de Armação, Atalaia e Caixa-Pregos.

Praia de Armação (Farias et al. 1985) A praia de Armação, localizada na costa atlântica de Salvador (Fig. 1), apresenta uma deriva litorânea cujo sentido se alterna ciclicamente ao longo do tempo, de uma ponta da praia para a outra. Como conseqüência, as duas extremidades mostram comportamentos inversos e revezados, com cada fase construtiva numa extremidade, de maneira geral, correspondendo a fase erosiva na outra, estando esse mecanismo relacionado à sazonalidade no regime de ventos local. Assim, quando está havendo erosão na extremidade $\mathrm{A}$ e conseqüente construção na extremidade B (Fig. 1), os ventos predominantes são de $\mathrm{SSE}$ e $\mathrm{S}$, e são responsáveis pela geração de frentes de onda que provocam deriva litorânea de sedimentos de A para B. Quando a deriva é inversa, correspondendo a uma erosão na extremidade B e a concomitante construção na extremidade A (Fig. 1), predominam ventos de E e NE, que induzem uma deriva nesse sentido. A par desses movimentos, existem também significativas trocas de materiais no sentido da face da praia para a antepraia e vice-versa.

Praia de Atalaia (Bittencourt et al. 1990a) A praia de Atalaia, localizada no município de Luís Correia, próximo à desembocadura do Rio Parnaíba, apresenta contiguamente à mesma um vasto campo de dunas ativas, por ela alimentado (Fig. 1). Ao longo de todo o ano, existe predominância de deriva litorânea de sedimentos no sentido de leste para oeste, provocada por frentes de onda geradas pelos ventos alíseos de E e NE. Ao lado disso, uma sazonalidade no regime de chuvas local cria condições, durante a estação da seca, para um intenso processo de deflação, que retira da face da praia para o campo de dunas adjacentes as frações granulométricas de areia fina e muito fina, restando uma concentração das frações mais grossas. Durante a época das chuvas, as mesmas inibem a remoção de areia da face da praia pela ação dos ventos, o que faz com que o sedimento praial, devido à ação

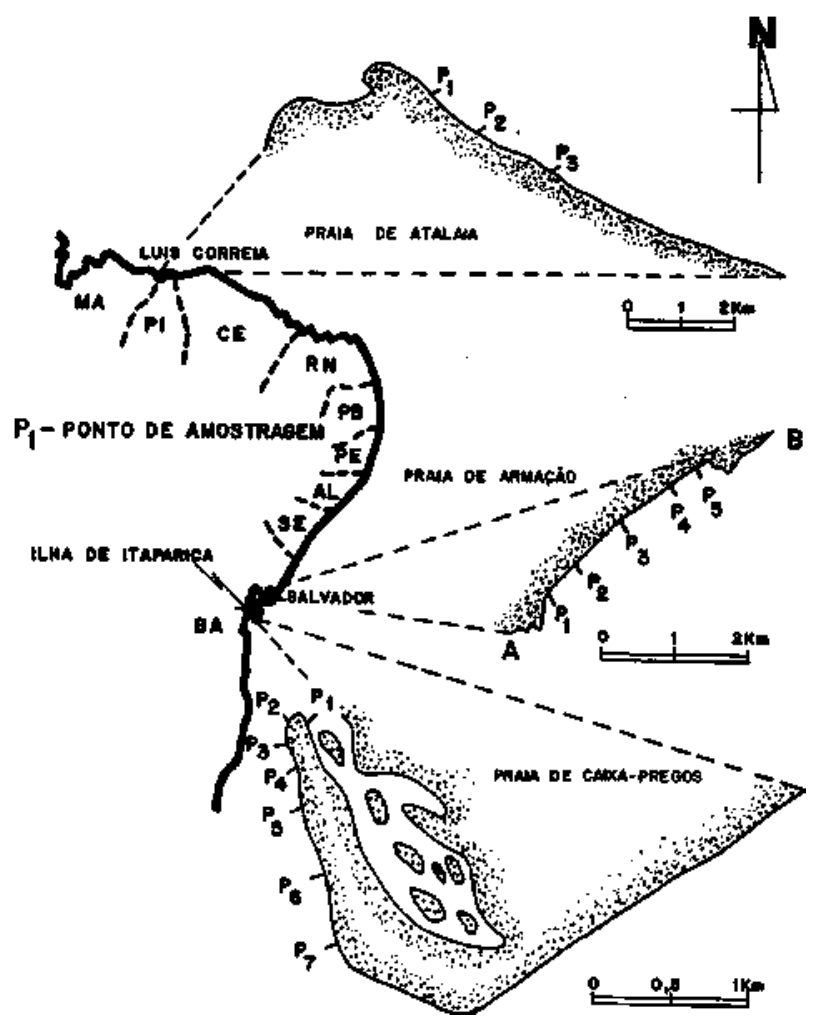

Figura 1 - Mapa de localização das praias de Armação, Atalaia e Caixa-Pregos mostrando os pontos de amostragem Figure 1 - Location map of the Armação, Atalaia and Caixa-Pregos beaches showing the sampl ing stations

das ondas construtivas que estão continuamente trazendo mediante a migração de barras, materiais predominantemente finos para a face da praia -, passe a apresentar, na sua maioria, constituição de areias fina a muito fina.

Praia de Caixa-Pregos (Bittencourt et al. 1990b) A praia de Caixa-Pregos está localizada num esporão situado na extremidade sul da ilha de Itaparica, defronte a Salvador (Fig. 1). O mecanismo de construção deste esporão está associado a um sentido único de deriva litorânea de sedimentos, a partir das praias situadas a leste do mesmo até a sua ponta, sendo desprezíveis as movimentações de materiais pela ação das ondas, tanto no sentido da antepraia para a face da praia e vice-versa, quanto da face da praia para o pós-praia, pela ação dos ventos.

\section{INDICAÇÕES DE SENTIDOS DE TRANSPORTE NAS PRAIAS DE ARMAČ̃̃O, ATALAIA E CAIXA - PREGOS}

Nas praias aqui analisadas foram coletadas amostras nos seguintes locais e faixas de tempo: a. praia de Armação - nos pontos PI, P2, P3, P4 e P5 (Fig. 1), durante o período de 157 $4 / 82$ a 20/9/83, num total de 42 amostras por ponto de coleta; b. praia de Atalaia - nos pontos PI, P2 e P3 (Fig. 1), durante o período de 3/8/85 a 23/4/86, num total de 17 amostras por ponto de coleta; c. praia de Caixa-Pregos - pontos PI, P2, P3, P4, P5, P6 e P7 (Fig. 1), no período de 25/6/87 a22/12/88, num total de 33 amostras por ponto de coleta. Essas amostras, após analisadas segundo a metodologia de McLaren (1981) descrita anteriormente, apresentaram uma série de indicações de sentidos de transporte entra as mesmas, que são mostradas nas tabelas 1, 2 e 3, respectivamente para as praias de Armação, Atalaia e Caixa-Pregos. Essas tabelas mostram, ainda, para as praias de Armação e de Atalaia, onde existem ventos que podem provocar derivas litorâneas com sentidos opostos, a 
Tabela 1 -Indicação de sentidos de transporte, ao longo do tempo, entre os diferentes pontos de amostragem na praia de Armação (Fig. 1). É também mostrada a proveniência dos ventos locais para cada dia de amostragem. Para o dia 27/5/82, por exemplo, para ventos provindos de E, que provocam deriva litorânea no sentido de B para A (Fig. 1), as indicações de sentidos de transporte mostraram que os sedimentos do ponto P5 podem ser considerados como fonte para os depósitos nos pontos P2 e P4, e os sedimentos do ponto P4 para os depósitos no ponto P2. Segundo McLaren (1981), uma amostra considerada como depósito é aquela que, no sentido do transporte, apresenta melhor selecionamento e assimetria mais positiva sobre as demais que a antecedem (consideradas como fonte)

Table 1 - Indications of transport directions during time, between different sampling points of Armação beach (Fig. 1). It is also shown the local wind provenance during each day of sampling. In 5/27/82, for instance, for easterly winds that induces a longshore drift from B to A (Fig. 1), the transport directions indications shows that sediments of point P5 can be considered as source, in a compatible manner, for deposits in points P2 and P4, and sediments of P4 for deposits in P2. According to McLaren (1981) a sample is considered a deposit if it presents a better sorting and more positive skewness when compared to those located updrift, which are considered to be

\begin{tabular}{|c|c|c|c|c|c|c|c|c|c|c|c|}
\hline DIA & FONTE & DEPOSITO & $\begin{array}{c}\text { ORIGEM } \\
\text { DOS } \\
\text { VENTOS }\end{array}$ & DIA & FONTE & DEROSITO & $\begin{array}{l}\text { ORHGEM } \\
\text { DOS } \\
\text { VENTOS }\end{array}$ & DLA & FONTE & DEPOSITO & $\begin{array}{l}\text { ORIGEM } \\
\text { DOS } \\
\text { VENTOS }\end{array}$ \\
\hline 15.04 .82 & PI & $\mathbf{P 3}$ & $\mathbf{s}$ & (12)09.82 & Ps & PI & E & & Ps & $\mathbf{P 2}$ & \\
\hline \multirow[t]{2}{*}{23.04 .82} & PI & $\mathbf{P 3}$ & $\mathbf{s}$ & & $P_{4}$ & PI.PL.p.ps & & 29.00 .83 & $\mathbf{P 2}$ & PI & SE \\
\hline & $P 2$ & $P P_{i} P S$ & & & P3 & PI & & & PJ & PI & \\
\hline $11,05.82$ & P5 & $\mathbf{P 3}$ & $E$ & $\operatorname{lol} 09.82$ & PI & $\mathbf{P}, \mathbf{P} 3, \mathbf{P 4}$ & SE & 1204.83 & PJ & P2. P3. P4 & SE \\
\hline \multirow[t]{2}{*}{$21.05: 82$} & P5 & $\mathbf{P I}, \mathbf{P 3}$ & $E$ & & 82 & P4 & & & P4 & $\mathbf{P} 2$ & \\
\hline & P3 & PI & & $24,04,82$ & $\mathbf{P 1}$ & P2. P3 & SE & & Ps & P3 & \\
\hline \multirow[t]{4}{*}{25.05 .82} & P5 & $\mathbf{P 2}, \mathbf{P d}$ & $\varepsilon$ & & P1 & $\mathbf{P 3}$ & & 120583 & PI & P3. P4. PS & SE \\
\hline & $\mathbf{P 4}$ & $\mathbf{P} 2$ & & & PS & P3 & & & $\mathbf{P 2}$ & P4, Ps & \\
\hline & $P 3$ & $\mathbf{P} \mathbf{2}$ & & 07.10.82 & PJ & P2 & E & & $\mathbf{P 3}$ & P4. PS & \\
\hline & $\mathbf{P 3}$ & P4,Ps & & & Pd & PI. P2, P3 & & & Ps & $\mathbf{P 4}$ & \\
\hline \multirow[t]{2}{*}{27.05 .82} & P5 & 12. P4 & $\mathbf{E}$ & 22.141282 & NENHUM & NDICAÇĀOO & & 26,15893 & PI & P5 & SE \\
\hline & P4 & $\mathbf{P 2}$ & & D6..11.82 & P4 & P2. Ps & NE & & $\mathbf{P} 2$ & PL.F & \\
\hline \multirow[t]{4}{*}{$04 \times 8.82$} & Ps & PI. $\mathbf{p} 2$ & $\mathbf{E}$ & 1211.82 & P4 & P1. PS & NE & & $\mathbf{P 3}$ & PI. P4, PS & \\
\hline & $\mathbf{P 4}$ & P1, P2 & & & P5 & $\mathbf{P 4}$ & & & $P_{4}$ & P5 & \\
\hline & $\mathbf{P 3}$ & P1, $\mathbf{P 2}$ & & 18.11 .82 & P1 & P3 & NE & $141 \times 8.83$ & PI & P2.M.PS & se \\
\hline & $\mathbf{P 4}$ & P5 & & & $\mathbf{P} 2$ & P1. P3 & & & $\mathbf{P} \mathbf{2}$ & PS & \\
\hline 18.06 .82 & $\mathbf{P 2}$ & $\mathbf{P 3}$ & SE & & P5 & $\mathbf{P 3}$ & & $22 \times 6,83$ & $\mathbf{P 2}$ & PS & $\mathbf{s}$ \\
\hline \multirow[t]{2}{*}{ 23.07.92 } & $\mathbf{P} \mathbf{1}$ & P2. Ps & SE & 24.11 .62 & $\mathbf{P 2}$ & P1,P3.PS & NE & & $\mathrm{P}^{3}$ & P4, PS & \\
\hline & $\mathbf{P 2}$ & P3 & & 16.01 .63 & $\mathbf{P 2}$ & $\mathbf{P 3}$ & E & & P4 & PS & \\
\hline \multirow[t]{4}{*}{29.17 .92} & P) & Ps & SE & 1401.83 & $\mathbf{P 2}$ & $\mathbf{P l}, \mathbf{P 4}$ & SE & 13.47 .83 & P1 & Ps & $\mathrm{SE}$ \\
\hline & $\mathbf{p}$ & Ps & & & $\mathbf{P 3}$ & P4 & & & P3 & P.5 & \\
\hline & $\mathbf{P 3}$ & $\mathbf{P I}, \mathbf{P}, \mathbf{P 4}, \mathbf{P S}$ & & 04.0283 & $\mathbf{P 2}$ & P4 & NE & & P4 & PS & \\
\hline & P4 & Ps & & 25,0083 & $\mathbf{P 3}$ & P2, PS & E & 27.07 .83 & P3 & P2. P4. P5 & SE \\
\hline \multirow[t]{3}{*}{6.08 .82} & Pt & P3. P4. PS & $\mathbf{S E}$ & & P4 & P2, Ps & & & PS & P2. 84 & \\
\hline & $\mathbf{P r}$ & Ps & & $04,03,83$ & $\mathbf{p} 2$ & P4 & E & $013,008,83$ & P1 & P2. P3, P4 & SE \\
\hline & $\mathrm{Pd}_{4}$ & Ps & & & 23 & P4 & & & $\mathbf{P} 2$ & P4 & \\
\hline 12.08 .82 & PS & P4 & SE & $10,03.93$ & PI & PS & SE & & $\mathbf{P 3}$ & P2. P4 & \\
\hline 19.08 .82 & P4 & P3, Py & E & & $P 2$ & P5 & & I(4.188.83 & $P 2$ & $\mathbf{P 3}$ & SE \\
\hline \multirow[t]{4}{*}{$27,08.82$} & PI & $\mathbf{P}$ & $E$ & & P4 & P2.PS & & 24,83 & PI & P2. P5 & SE \\
\hline & PS & $\mathrm{P2} \cdot \mathrm{P3}$ & & 1703.23 & $\mathbf{P l}$ & $\mathbf{P 2}$ & E & & $\mathrm{PA}^{4}$ & $\mathbf{P S}$ & \\
\hline & $\mathbf{P 4}$ & $\mathbf{P 3}$ & & & $\mathbf{P 3}$ & P1.P2.PS & & 2009.83 & PI & P5 & SE \\
\hline & & & & & & & & & P3 & $\mathbf{P 2}$ & \\
\hline
\end{tabular}

proveniência dos mesmos para cada dia de monitoramento, a fim de se poder confrontar o sentido da deriva com a indicação de tendência de transporte.
Para a praia de Atalaia são apresentadas, segundo Bittencourt et al. (1990 a), as épocas do ano com e sem deflação da areia da face da praia para os campos de dunas contíguos (Tab. 2). 
Tabela 2 - Indicações de sentidos de transporte, ao longo do tempo, entre os diferentes pontos de amostragem na praia de Atalaia (Fig. 1). E mostrada a proveniencia dos ventos locais para cada dia de amostragem e as fases com e sem deflação de areia da face da praia. Para entendimento da tabela ver exemplo na legenda da tabela 1

Table 2 - Indications of transport directions during time, between different sampling points of Atalaia beach (Fig. 1). It is also shown the local wind provenance during each day of sampling as well as the phases with and without sand deflation from the beach face. For understanding the table see example in caption of table I

\begin{tabular}{|c|c|c|c|c|}
\hline DIA & FONTE & DEPOSITO & $\begin{array}{l}\text { ORIGEM } \\
\text { DOS } \\
\text { VENTOS }\end{array}$ & \\
\hline 03.08 .85 & $\mathbf{P 2}$ & PI & $\mathbf{E}$ & \multirow{9}{*}{$\begin{array}{c}\text { FASE } \\
\text { COM } \\
\text { DEFLACA }\end{array}$} \\
\hline 04.09 .85 & PI & $P_{2}, P_{3}$ & $\mathbf{E}$ & \\
\hline 17.09 .85 & \multicolumn{2}{|c|}{ NENHUMA INDICACÃO } & & \\
\hline 01.10 .85 & \multicolumn{2}{|c|}{ NENHUMA INDICAÇĀO } & & \\
\hline 16.10 .85 & \multicolumn{2}{|c|}{ NENHUMA INDICAÇÃO } & & \\
\hline 23.10 .85 & PI & $P_{2}, P_{3}$ & $\mathrm{~F}$ & \\
\hline \multirow[t]{2}{*}{08.11 .85} & PI & P2 & NE & \\
\hline & P3 & $\mathbf{P 2}$ & & \\
\hline 19.11 .85 & P3 & $\mathbf{P 2}$ & NE & \\
\hline 10.12 .85 & P3 & Pl & NE & \multirow{11}{*}{$\begin{array}{c}\text { FASE } \\
\text { SEM } \\
\text { DEFLACA }\end{array}$} \\
\hline 18.12 .85 & P3 & $\mathbf{P 2}$ & WE & \\
\hline 09.01 .85 & P3 & P2, P1 & $\mathrm{NE}$ & \\
\hline 04.02 .85 & Pl & $\mathrm{P2}$ & NE & \\
\hline 13.02 .86 & P3 & P2, Pl & NE & \\
\hline 26.02 .86 & $\mathbf{P I}$ & P3 & NE & \\
\hline 14.03 .86 & P3 & P2, PI & NE & \\
\hline \multirow{3}{*}{03.04 .86} & $\mathbf{P t}$ & $\mathbf{P} 2$ & & \\
\hline & PI & $\mathbf{P 2}$ & $\mathbf{E}$ & \\
\hline & P3 & $\mathbf{P 2}$ & & \\
\hline 23.04 .86 & P3 & P2 & $\mathbf{E}$ & \\
\hline
\end{tabular}

Sobre as indicações de sentidos de transporte, elas são apresentadas mediante a identificação, para cada dia, das amostras que podem ser consideradas como fonte para outras que, no sentido do transporte, são consideradas como depósito. Assim, por exemplo, na tabela 1, observa-se que, no dia 27/5/82, na praia de Armação, para ventos provindos de leste, os sentidos de transporte entre as amostras são compatíveis com a deriva litorânea provocada pelas frentes de onda geradas por tais ventos ao longo da praia, no sentido de P5 para PI (Fig. 1). Pelas tabelas 1, 2 e 3, constata-se que varia bastante o número de indicações de sentidos de transporte por dia, havendo casos em que aparece, para um número máximo possível de $1 \mathrm{O}$ indicações corretas, como na praia de Armação (Fig. 1), somente uma única indicação (por exemplo, dia 157 4/82, Tab. 1). Pode ser observado que num mesmo dia podem aparecer indicações de sentidos de transporte opostas. Isso pode ser verificado que no dia $25 / 6 / 87$, na praia de CaixaPregos (Tab. 3), onde, para um número máximo possível de 21 indicações corretas, existem 11 indicações compatíveis (como mencionado anteriormente, no sentido geral de P7 para P1, Fig. 1) e três indicações incorretas. Nesses casos, como o número efetivo de indicações de sentidos de transporte aponta para o sentido da deriva litorânea, são computadas como corretas. Por outro lado, existem situações em que prevalecem indicações de sentidos de transporte que são flagrantemente contrárias à deriva litorânea existente, como é o caso do dia 4/3/83, na praia de Armação, onde - para ventos provindos de $\mathrm{E}$ (Tab. 1), que geram frentes de onda que induzem uma deriva litorânea no sentido de P5 para P1 (Fig. 1) -, as indicações apontam em sentido contrário (Tab. 1). Nesses casos, as indicações são computadas como incorretas. Por fim, quando, em algum dia, não há uma predominância de indicações num determinado sentido, como no dia 8/11/ 85, na praia de Atalaia (Tab. 2), esse resultado, para fins estatísticos, é computado semelhantemente aos dias em que não há nenhuma indicação, como o dia 22/10/82, na praia de Armação (Tab. 1).

A tabela 4 mostra, porcentualmente, para cada praia analisada, o resumo das indicações de sentidos de transporte apresentadas nas tabelas 1, 2 e 3, segundo os critérios anteriormente mencionados. Por essa tabela, observa-se que existe marcante predominância de dias com indicações corretas. Exceção é feita à praia de Atalaia na época em que há deflação de areia da face da praia para o pós-praia, quando, segundo os critérios acima citados, predominam dias em que não há nenhuma indicação de sentido de transporte. Das três praias, a praia de Caixa-Pregos é a que apresenta um maior percentual de dias com indicações corretas, seguida das praias de Armação e de Atalaia (Tab. 4).

DISCUSSÃO E CONCLUSÕES Os resultados obtidos com a aplicação do método de análise de sentidos de transporte de McLaren (1981) nas amostras coletadas nas praias analisadas neste trabalho, de maneira geral, correlacionam-se bem com o processo geológico predominante nas mesmas. Exceção é feita à praia de Atalaia, na estação seca, quando há remoção de areia da face da praia para o pós-praia (Tab. 4). Assim, para uma praia que durante todos os dias de monitoramento apresentou sempre deriva litorânea no sentido de P3 para PI (Tab. 2 e Fig. 1), apenas 25\% das indicações de sentidos de transporte apresentaram resultados coincidentes, sendo o restante das medidas constituídas de $25 \%$ de indicações em sentido contrário ao da deriva e de $50 \%$ sem nenhuma indicação (Tab. 4). Tal discrepância pode estar relacionada ao fato de que o processo de deflação não é uniforme ao longo de toda a praia, o que implica em que a retirada das frações granulométricas finas da face da praia, sob a ação do vento, é feita em diferentes quantidades ao longo da praia. As variações topográficas na superfície de uma praia, como as causadas na praia de Atalaia pelas barras que estão continuamente migrando da antepraia para a face da praia (Bittencourt et al 1990a), podem provocar dificuldades ao movimento das partículas pela ação do vento. Quando a superfície da praia é plana, o movimento da areia se processa de uma forma efetiva e contínua (Short \& Hesp 1982). Todavia, qualquer variação no gradiente praial altera o movimento das partículas, por criarem zonas de turbulência próximas a essas áreas e, dessa maneira, influírem na velocidade e na direção da movimentação dos grãos, bem como no volume de material transportado (Svasek \& Terwindt 1974, Bowen \& Lindley 1977).

Os dias em que foram obtidos resultados anômalos ou sem nenhuma indicação, nas praias de Armação e CaixaPregos, e na praia de Atalaia na fase sem deflação, podem estar relacionados a problemas da técnica de amostragem. $\mathrm{O}$ aspecto fundamental desses problemas é que a metodologia de McLaren (1981), segundo o qual um sedimento deve ser comparado com outro para prover uma compreensão do processo sedimentar atuante, implica na coleta de amostras que sejam efetivamente representativas dos dois corpos de 
Tabela 3 - Indicações de sentidos de transporte, ao longo do tempo, entre os diferentes pontos de amostragem na praia de CaixaPregos (Fig. 1). Os ventos atuantes no local promovem sempre deriva litorânea no sentido do ponto P1 para P1 (Fig. 1). Para entendimento da tabela ver exemplo na legenda da tabela 1

Table 3 - Indications of transport directions during time, between different sampl ing points of Caixa-Pregos beach (Fig. 1). The local wind action all ways induces a longshore drift directed from point P7 to P1 (Fig. 1). For understanding the table see example in caption of table 1

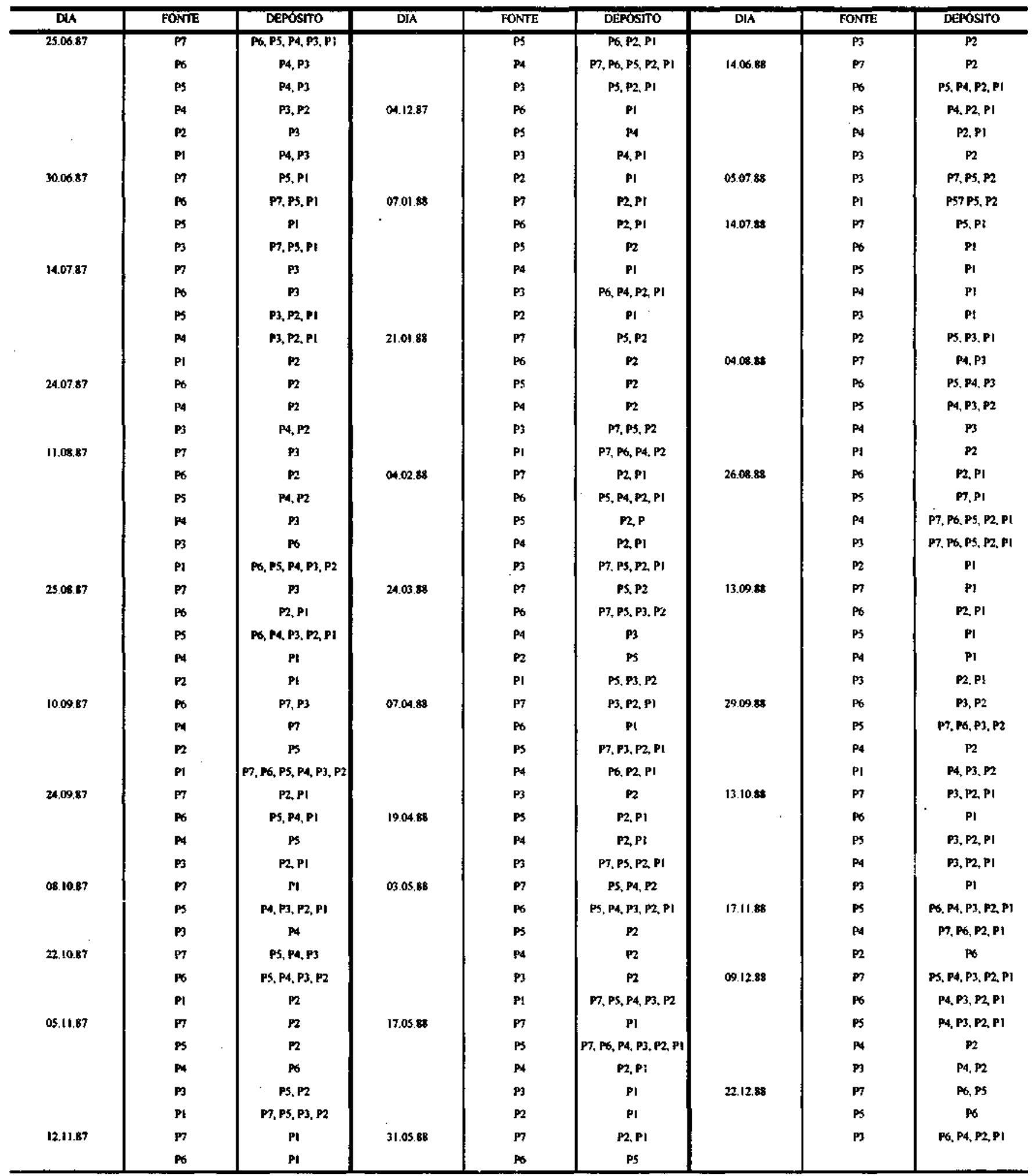

sedimentos que estão sendo comparados. A questão que assoma em importância está relacionada ao que se deve entender por amostragem representativa para sedimentos da face da praia. As técnicas rotineiras de coleta de amostras na face da praia, mesmo no caso deste trabalho, quando da coleta de amostras representativas dos dois primeiros centímetros superficiais do sedimento, resultam quase sempre numa mistura de algumas lâminas sedimentares do depósito praial. Embora Griffiths (1967) lembre que a amostra mais representativa é a que contém número de lâminas suficiente para 
Tabela4-Números de dias, em porcentagens, com indicações de sentidos de transporte corretas, incorretas e sem nenhuma indicação, para as praias de Armação, Atalaia e CaixaPregos

Table 4 - Number of days, in percentage, indicating correct and incorrect transport directions or without indications, from Armação, Atalaia and Caixa-Pregos beaches

\begin{tabular}{|c|c|c|c|c|}
\hline 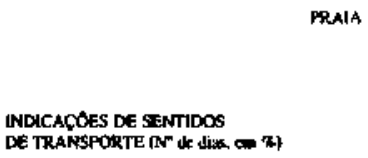 & ARMAÇĀO & 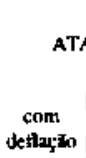 & $\operatorname{sem}_{\text {deflacipin }}$ & $\begin{array}{l}\text { CAIXA } \\
\text { PREGOS }\end{array}$ \\
\hline INDICAÇOES CORRETAS & 64 & 25 & 66 & 74 \\
\hline NENHUMA INDICAÇAO & 16 & s) & 12 & 9 \\
\hline INDTCACOES JNCORRETAS & 16 & 25 & 22 & 17 \\
\hline
\end{tabular}

prover distribuição granulométrica representativa de todo o depósito, resta sempre a questão sobre o número de lâminas suficiente para tanto. A rigor, para fins de análise de sentidos de transporte, uma amostragem representativa deveria ser feita ao longo de uma única lâmina, porque como apontam Sonu (1972) e Emery \& Stevenson (1950), ela seria representativa de processo uniforme de deposição. Nesse sentido, como cada lâmina representa uma condição particular dos movimentos de saca e ressaca, os quais estão continuamente mudando, as suas características granulométricas, normalmente, como aponta McLaren (1981), são o resultado de condições bem locais para uma efetiva comparação em escala mais regional. Por isso, para fins de análise de sentidos de transporte, a amostragem ideal na face da praia seria aquela feita ao longo de uma mesma lâmina, com intervalos de amostragem que seriam limitados pela própria dimensão das lâminas sedimentares na face da praia que, normalmente, segundo Thompson (1937), nãoexcede 30 mem praias atuais. Tal técnica de amostragem só poderia ser considerada conveniente para pequenos trechos praiais, uma vez que a mesma implica na abertura de trincheiras longitudinais à face da praia, a fim de que se possa ter a certeza de se estar amostrando sempre a mesma lâmina do sedimento praial. Em trechos praiais relativamente extensos, como os analisados neste trabalho, o ato de coleta nessas circunstâncias demandaria esforços muito grandes. Essas considerações sobre erro na técnica de amostragem são pertinentes às amostras coletadas na praia de Atalaia durante a fase de deflação.

Outra consideração que deve ser feita em relação aos resultados obtidos neste trabalho, incluindo-se aqueles sobre a praia de Atalaia na fase de deflação, refere-se ao fato de que a praia de Caixa-Pregos foi a que apresentou o maior percentual de indicações corretas do sentido da deriva (74\%) seguida, em porcentagens quase idênticas, pelas praias de Armação (68\%) e de Atalaia (66\%) (na fase sem deflação). É possível pensar que esse fato esteja relacionado à utilização de diferentes fórmulas para o cálculo dos parâmetros estatísticos empregados neste trabalho. Tal circunstância, todavia, não deve ser considerada como um entrave para a comparação dos resul- tados, uma vez que, como reconhecem Duane (1964), Friedman (1967) e Emery (1978), ambas as fórmulas aqui utilizadas fornecem resultados que concordam entre si. O maior percentual de indicações corretas nessa praia provavelmente está relacionado tanto às características fisiográficas particulares do esporão de Caixa-Pregos, quanto ao número de amostras nele coletadas. No primeiro caso, pelo fato dessa feição geomórfica ter a sua dinâmica de sedimentação essencialmente ligada à deriva litorânea, diferentemente das demais outras praias, onde intervém outros mecanismos de transporte, as variações das características lexturais do sedimento praial de um ponto para outro de amostragem devem ser mais uniformes, o que deve favorecer a aplicação do método de análise de tendência de McLaren (1981). Um exemplo significativo de como a intervenção de mais de um mecanismo de transporte na face da praia pode vir a mascarar os resultados da análise de tendência, para os dados deste trabalho, é mostrado pela praia de Atalaia na fase com deflação. No segundo caso, o problema está relacionado ao princípio probabilistic de que, quanto maior o número de medidas, tanto maior o grau de precisão das mesmas (Griffitts 1967). Nesse sentido, nas praias de Caixa-Pregos foi coletado um maior número de amostras por diadoque nas demaisoutras. Sobre a aplicabilidade do método de McLaren (1981) para a definição, por exemplo, do sentido da deriva litorânea que prevaleceu durante a deposição de um corpo praial antigo que, porventura, esteve sujeito a esse tipo de transporte, devem ser feitas considerações. Em primeiro lugar, em corpos sedimentares do passado há sempre a possibi lidade de que os processos diagenéticos tenham alterado a composição granulométrica original (Friedman 1962, Hails \& Hoyt 1969), o que poderá influir negativamente na análise de sentidos de transporte segundo o método aqui discutido. Em segundo lugar, podem existir dificuldades, pela inexistência de trechos de afloramentos contínuos e extensos que sejam suficientes para coleta representativa de todo o corpo praial, na forma de amostras cronocorrelatas, para que conforme a metodologia de McLaren (1981), seja possível comparar as características granulométricas de amostras relacionadas ao mesmo evento sedimentar. Acresce ainda o fato de que a amostragem deve ser feita ao longo de toda a sucessão vertical do corpo, para que se possa identificar as eventuais reversões da deriva litorânea que tenham eventualmente ocorrido e deixado registro durante a deposição praial. Por fim, vale ressaltar que, no caso de épocas de remoção de areia da face da praia para o pós-praia, pela ação dos ventos, durante a formação do depósito praial antigo, como ocorreu praia de Atalaia, o método de McLaren (1981) não deverá funcionar tão bem quanto em situações em que inexiste tal processo. Todavia, mesmo em tais circunstâncias, a praia de Atalaia mostra, no global, considerando-se apenas os casos em que houve indicação, $65 \%$ de indicações corretas e $35 \%$ de incorretas.

Agradecimentos Os autores deixam aqui expressos os seus agradecimentos ao prof. Ordônio Moita P, pela coleta das amostras na praia de Atalaia, ao prof. José M. L. Dominguez, pela versão do resumo para o inglês, e aos bolsistas de Iniciação Científica dó CNPq, Aírton C. Matos, P, Antônio P. Menezes e Jorge P. Cid, pelos cálculos computacionais.

\section{REFERENCIAS BIBLIOGRÁFICAS}

BITTENCOURT, A.C.S.P ; DOMINGUEZ, J.M.L; MOITA, O., P 1990a. Variações texturais induzidas pelo vento nos sedimentos da face da praia (Praia de Atalaia - Piauí). Rev. Bras. Geoc., 20:201 -207.

BITTENCOURT, A.C.S.P.; FARIAS, F.F.; VILAS BOAS, G.S. 1990b. Influência da deriva litorânea no desenvolvimento do esporão de Caixa-Pregos (Baía de Todos os Santos/BA). Rev. Bras. Geoc, 20:197-200.

BITTENCOURT, A.C.S.P.; FARIAS, F.F.; ZANINI, A. Jr. 1987. Reflexos das variações morfodinâmicas praiais nas características texturais dos sedimentos da praia de Armação, Salvador, Bahia. Rev. Bras. Geoc., 17:276-282.
BOWEN, A.J. \& LINDLEY, D. 1977. A wind-tunnel investigation of wind turbulence characteristics close to the ground over various escarpment shapes. Boundary-Layer Meteorology, 12:259-271.

DUANE, D.B. 1964. Significance of skewness in recent sediments, Western Pamlico Sound, North Carolina. J. Sed. Petrol. 34:864-874.

EMERY, K.O. 1978. Grain size in laminae of beach sand. J. Sed. Petrol., 48:1203-1212

EMERY, K.O. \& STEVENSON, R.E. 1950. Laminated beach sand. J. Sed. Petrol., 20:220-223. 
FARIAS,F.F.;BITTENCOURT,A.C.S.P.;ZAN1NI,A., Jr.;DOMINGUEZ, J.M.L. 1985. Variações temporaiseespaciaisnadinâmicade sedimentação da praia de Armação - Salvador/B A. Rev. Bras. Geoc., 15:48-54

FOLK, R.L. \& WARD, W.C. 1957. Brazos River bar: astudy in the significance of grain size parameters. J. Sect. Petrol., 27:3-26.

FRIEDMAN, G.M. 1961. Distinction between dune, beach, and river sands fromtheirtextural characteristics. $J$ Sed Petrol, 31:514-529.

FRIEDMAN, G.M.I 962. On sorting coefficients and the lognormal ity of the grain size distribution of sandstones. J.Geol., 70:737-753.

FRIEDMAN, G.M. 1967. Dynamic processes and statistical parameters compared for size frequency distribution of beach and river sands. J. Sed. Petrol. 37:327-354.

GRIFFITHS, J.C. 1967. Scientific method in analysis of sediments. New York, McGraw-Hill Company. 508 p.

HAILS, J.R.\&HOYT,J.H. 1969. The significance and limitationsof statistical parameters for distinguishing ancient and modem sedimentary environments ofthe lower Georgia coastal plain. J. Sed. Petrol., 39:559-580.
McLAREN, P. 1981. An interpretation of trends in grain size measures. J. Sed. Petrol., 51:611-624.

SHORT, A.D. \& HESP, P.A. 1982. Wave, beach and dune interactions in southeastern Australia. Mar.Geol.,48:259-284.

SONU, C.J. 1972. Bimodal composition and cyclic characteristics of beach sediment in continuously changing profiles. J.Sed.Petrol.,42:\&52-\&51.

SVASEK,J.N.\&TERWINDT,.H.J. 1974. Measurement of sand transport by wind on a natural beach. Sedimentology, 21:311 -322.

THOMPSON, W.0.1937. Original structures of beaches, bars, and dunes. Bull. Geol.Soc.A m., 48:723-752.

MANUSCRITO A707 Recebido em 3 de setembro de 1991 Revisão do autor em 2 de março de 1992 Revisão aceita em 5 de março de 1992 